\title{
Demyelinating Lesion Isolated to the Lateral Geniculate Nucleus
}

\author{
Joanna Suski ${ }^{*}$ and Myla Goldman
}

Department of Neurology, University of Virginia, Charlottesville, USA

*Corresponding author: Joanna Suski, Department of Neurology, University of Virginia, Charlottesville, VA 22908-3094, USA, Tel: 434-924-2706; Fax: 434-243-5420; E-mail: jls8fx@virginia.edu

Received date: Mar 21, 2014, Accepted date: Apr 23, 2014, Published date: Apr 28, 2014

Copyright: $\odot 2014$ Suski J, et al. This is an open-access article distributed under the terms of the Creative Commons Attribution License, which permits unrestricted use, distribution, and reproduction in any medium, provided the original author and source are credited.

\begin{abstract}
Symptomatic retrochiasmatic lesions due to demyelinating disease are rare in multiple sclerosis. The most common cause of visual disturbance in multiple sclerosis is optic neuritis which typically presents with pain around the eye that worsens with movement, dyschromatopsia and monocular decreased visual acuity. We describe a patient who presented with bilateral blurry vision who was found to have a left congruous homonymous sectoranopia due to a right lateral geniculate nucleus demyelinating lesion.
\end{abstract}

Keywords: Multiple Sclerosis; Optic neuritis; Visual loss; Visual field

\section{Introduction}

A 45 year old right-handed woman with a history of relapsing remitting multiple sclerosis (RRMS) with associated left optic neuritis in 2000, presented with subacute bilateral blurry vision of two days duration. She had occasional residual blurry vision in her left eye from the 2000 episode of optic neuritis, but had normal right eye vision at baseline. She did not have pain with eye movement, double vision or decreased color vision. She was recently started on gabapentin two weeks ago and had no other new medication changes.

On examination, her vital signs were normal. On mental status testing, she was alert and oriented. Her cranial nerve examination demonstrated an old left relative afferent papillary defect (RAPD). Her left optic disc demonstrated pallor. Her visual acuity was 20/20 in both eyes. Her extraocular movements were normal and without evidence of internuclear ophthalmoplegia (INO). Her visual fields were normal to confrontation. She had no facial weakness with normal facial sensation. On motor exam, she had full strength throughout. Her reflexes were symmetric and normal throughout. Plantar reflexes were downgoing. Sensation was normal. Finger to nose and heel to shin was without dysmetria. Gait was normal with negative Romberg.

The most common visual manifestation of MS is optic neuritis, which would be unlikely in our patient unless she had bilateral optic neuropathy. Our patient did not describe the typical symptoms of optic neuritis as she had absence of painful eye movements, dyschromatosia, and no new afferent papillary defect while having normal visual acuity. Medication side effects can be the culprit, but in our case, gabapentin was discontinued as this was newly started without any resolution in her symptoms.

Due to her complaint of bilateral visual loss without a visual field defect found on bedside exam, she was referred to ophthalmology. The ophthalmological examination revealed normal visual acuity of 20/20 in both eyes on far testing. Pupils were equal, round and reactive to light with a left RAPD. Visual fields were full to confrontation. Her fundoscopic exam showed right disc temporal sloping and left disc pallor. She had optical coherence tomography with her right retinal nerve fiber layer measuring $105 \mu \mathrm{m}$ and her left eye measuring $49 \mu \mathrm{m}$, which is evidence of a previous optic neuritis in the left eye. Her humphrey visual field testing demonstrated a left sectoranopia, congruous homonymous hemianopsia that respected the midline (Figure 1).
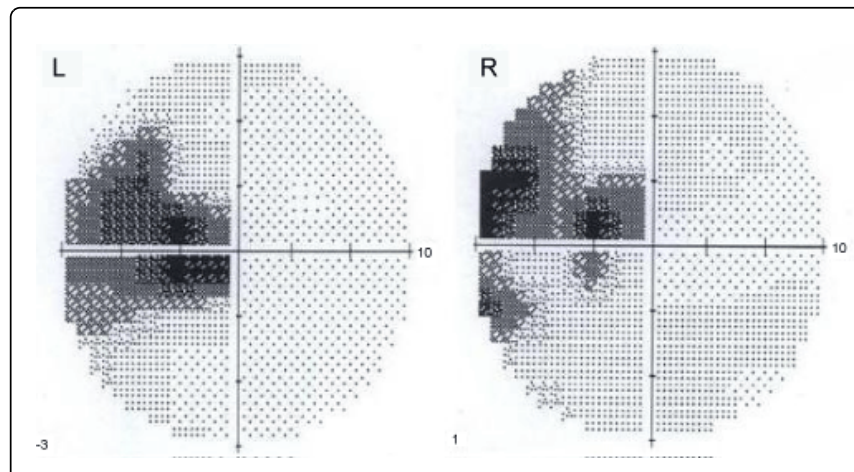

Figure 1: Humphrey Visual Field Testing shows a left congruous homonymous sectoranopia.

The left congruous homonymous sectoranopia suggested a retrochiasmal lesion so MRI brain with and without contrast was done to evaluate for stroke, tumor, cerebral abscess, demyelination, infectious etiologies, aneurysms or vascular malformations in the right hemisphere affecting the optic radiations [1].

The contrasted MRI demonstrated a new $7 \mathrm{~mm}$ enhancing lesion, without restricted diffusion, in the right lateral geniculate nucleus of the thalamus.

\section{Discussion}

The most common cause of subacute visual loss in MS patients is acute optic neuritis occurring in over $50 \%$ of patient [1]. Isolated visual loss due to demyelination in the optic tracts or cerebral hemispheres is rare in multiple sclerosis. Our patient presented with painless, sub-acute progressive vision loss in both eyes. Due to previous pre-chiasmal disease, our patient failed to fully appreciate the bilateral or field specific nature of her vision symptoms and the 
Citation: Suski J, Goldman M (2014) Demyelinating Lesion Isolated to the Lateral Geniculate Nucleus. J Neurol Neurophysiol S12: S12-014. doi:

cursory exam in the clinic did not detect a sectoranopsia. This case illustrates the importance of formal visual field testing in the diagnostic workup when there are complaints of bilateral visual loss in patients with MS (Figure 2).

Homonymous hemianopia $(\mathrm{HH})$ is usually caused by stroke, trauma, tumor, and rarely by demyelinating lesions. In a retrospective review of 904 cases by Zhang, they found demyelination to be the cause of $\mathrm{HH}$ in $1.4 \%$ of the case and lesions involving the lateral geniculate nucleus in $1.2 \%$ of the cases [2]. Sectoranopias have been described more robustly in vascular lesions affecting the lateral posterior choroidal artery or anterior choroidal artery. Vascular lesions in the lateral geniculate nucleus are rare due to the dual artery supply but when they do occur they produce either a sector sparing homonymous hemianopsia or homonymous sectoranopia. An anterior choroidal artery occlusion produces a sector sparing homonymous hemianopsia, whereas a lateral posterior choroidal artery occlusion produces a homonymous sectoranopia [3].

Although symptomatic homonymous field deficits are rare in patients with multiple sclerosis, asymptomatic homonymous field deficits can occur and may be more common than expected. The Optic Neuritis Treatment Trial $8.9 \%$ of the 488 patients had asymptomatic homonymous hemianopsia at their one year follow up [4]. In another study done by Plant, they reported 18 patients out of 2,000 had symptomatic retrochiasmal lesions found by MRI [5]. A common theme was that all the lesions were large. Small lesions in the brain are likely not symptomatic since there is more area per volume and fibers follow a curvilinear course. Fibers in the optic nerve are more concentrated with less space for the edema to spread causing a secondary compressive component, therefore causing symptoms more frequently [5].

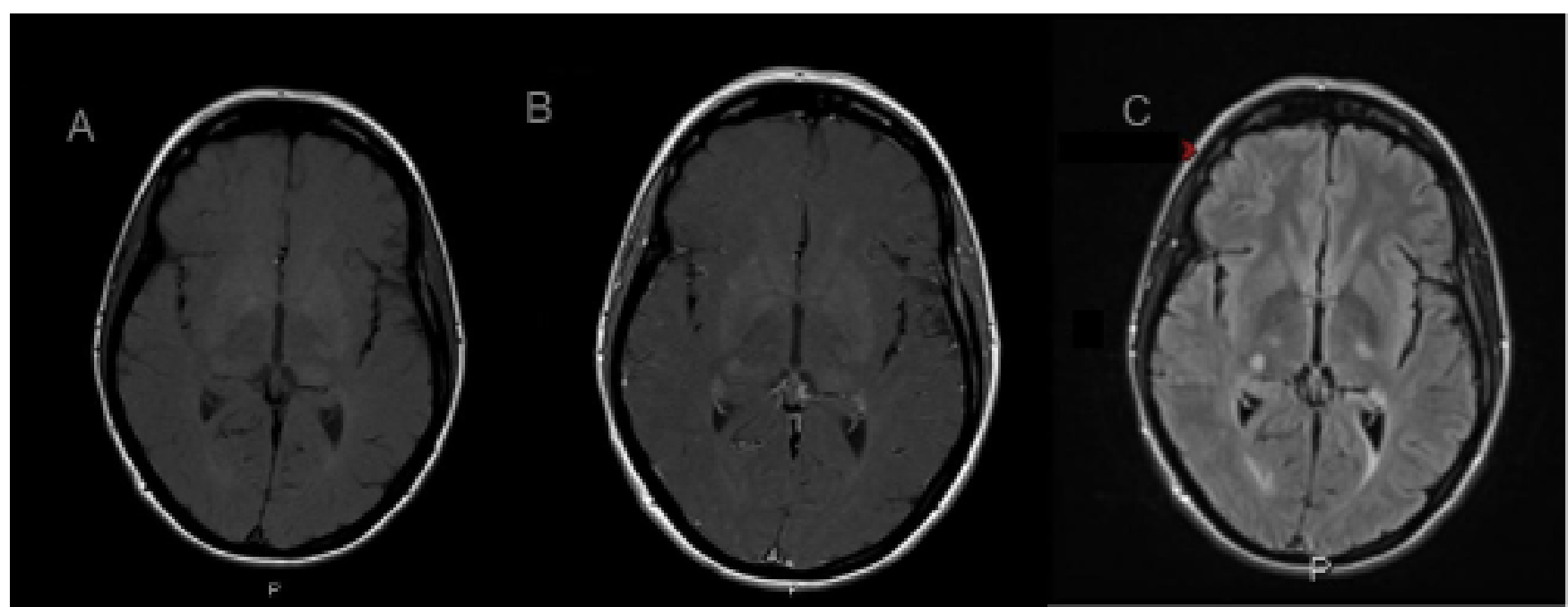

Figure 2: (A) MRI axial brain T1 normal. (B) MRI axial brain T1 post contrast, shows a $7 \mathrm{~mm}$ contrast enhancing lesion within the posterior right lateral thalamus. (C) MRI T2 axial brain fluid-attenuated inversion recovery (FLAIR) shows a 7 mm hyperintensity within the posterior right lateral thalamus.

In summary, our patient presented with subacute bilateral visual loss and was found to have a left homonymous sectoranopia due to a right geniculate nucleus demyelinating lesion. Her sectorectopia was not picked up on bedside visual field testing, hence stressing the importance of formal visual field testing. Although it is rare to see symptomatic retrochiasmatic demyelinating lesions in MS, it should be kept in the differential to ensure prompt diagnosis and treatment.

\section{References}

1. Graves J, Balcer LJ (2010) Eye disorders in patients with multiple sclerosis: natural history and management. Clin Ophthalmol 4: 1409-1422.
2. Zhang X, Kedar S, Lynn MJ, Newman NJ, Biousse V (2006) Homonymous hemianopias: clinical-anatomic correlations in 904 cases. Neurology 66: 906-910.

3. Luco C, Hoppe A, Schweitzer M, Vicuña X, Fantin A (1992) Visual field defects in vascular lesions of the lateral geniculate body. J Neurol Neurosurg Psychiatry 55: 12-15.

4. Keltner JL, Johnson CA, Spurr JO, Beck RW (1994) Visual field profile of optic neuritis. One-year follow-up in the Optic Neuritis Treatment Trial. Arch Ophthalmol 112: 946-953.

5. Plant GT, Kermode AG, Turano G, Moseley IF, Miller DH, et.al. (1992) Symptomatic retrochiasmal lesions in multiple sclerosis - clinical features, visual evoked potentials, and magnetic-resonance imaging. Neurology 42: 68-76. 